\title{
Incidence and Predictors of Transcatheter Aortic Valve Replacement Device Emboligenic Matter Detected By Transesophageal Echocardiograph
}

\author{
Masaki Yashige ( $\nabla$ shige912@koto.kpu-m.ac.jp ) \\ Graduate School of Medical Science Kyoto Prefectural University of Medicine https://orcid.org/0000-0002-4143- \\ $138 \mathrm{X}$
}

Kan Zen

Department of Cardiovascular Medicine, Graduate School of Medical Science, Kyoto Prefectural University of Medicine

\section{Shunsuke Nakamura}

Graduate School of Medical Science, Kyoto Prefectural University of Medicine

\section{Tomotaka Fujimoto}

Graduate School of medical Science, Kyoto Prefectural University of Medicine

\section{Kazuaki Takamatsu}

Graduate School of Medical Science, Kyoto Prefectural University of Medicine

\section{Nobuyasu Ito}

Graduate School of Medical Science, Kyoto Prefectural University of Medicine

\section{Yoshito Kadoya}

Graduate School of Medical Science, Kyoto Prefectural University of Medicine

\section{Michiyo Yamano}

Graduate School of Medical Science, Kyoto Prefectural University of Medicine

\section{Tetsuhiro Yamano}

Graduate School of Medical Science, Kyoto Prefectural University of Medicine

\section{Takashi Nakamura}

Graduate School of Medical Science, Kyoto Prefectural University of Medicine

Hitoshi Yaku

Kyoto Prefectural University of Medicine

\section{Satoaki Matoba}

Graduate School of Medical Science, Kyoto Prefectural University of Medicine

\section{Research Article}

Keywords: aortic stenosis, transesophageal echocardiography, transcatheter aortic valve replacement, ischemic stroke, embolization

Posted Date: November 12th, 2021

DOI: https://doi.org/10.21203/rs.3.rs-1049933/v1 
License: (1) This work is licensed under a Creative Commons Attribution 4.0 International License. Read Full License 


\section{Abstract}

Purpose: During transfemoral (TF) or transcatheter aortic valve replacement (TAVR), transesophageal echocardiography (TEE) sometimes reveals an unexpected mobile membranous mass on the catheter tip within the proximal part of the descending thoracic aorta. Such mobile masses may cause critical embolic events if the TAVR device advances into the ascending aorta in the absence of preventive measures. The purpose of this study was to investigate the incidence and predictors of emboligenic matter (EM) during TAVR, impact of EM on the procedure, and incidence of symptomatic ischemic stroke post procedure.

Methods: Among 436 consecutive patients who underwent TF-TAVR, 407 were evaluated in this study. The primary end point was incidence of symptomatic ischemic stroke within $24 \mathrm{~h}$ post procedure, while taking appropriate preventive measures. Incidence of EM, factors associated with EM and the impact of EM on the procedure, were also investigated.

Results: Among the 407 cases, 15 cases (3.7\%) of EM were identified but no ischemic stroke occurred in the EM (+) group ( $0 \%$ vs. $2.04 \%, p=1.00)$. In the EM (+) group, a self-expandable valve was used in all 15 cases $(100 \%$ vs. $42.6 \%, p<0.0001)$ while 14 cases used a CoreValve's InLine sheath system initially (93.3\% vs. $27.3 \%, p<0.0001)$. CoreValve's InLine sheath system usage was the only independent predictor of EM.

Conclusion: The CoreValve's InLine delivery system was identified as a predictor of EM during TF-TAVR, but symptomatic ischemic stroke was avoided while taking appropriate embolization preventive measures.

\section{Introduction}

The number of transcatheter aortic valve replacements (TAVR), mostly via transfemoral (TF) access, is increasing and the procedure has been simplified due to increased physician experience and TAVR device system refinement. Currently, the possibility of TF-TAVR under only local anesthesia or conscious sedation appears appealing and transesophageal echocardiography (TEE) is less relevant with this minimalistic approach. Several studies have demonstrated that TF-TAVR under local anesthesia or conscious sedation without TEE is as safe as that performed under TEE guidance and general anesthesia [1, 2]. Recently, the proportion of TAVR performed using conscious sedation has increased, to $64.1 \%$ in the United States for instance, where it was associated with improved clinical outcomes compared with general anesthesia [3].

We also reported an unexpected mobile membranous mass on the catheter tip within the proximal part of the descending thoracic aorta revealed by intraoperative TEE during TF-TAVR, which was successfully completed without serious complication after reinsertion of the device through an additional outer sheath [4] (Supplemental Video 1). Such mobile masses attached to the catheter tip may cause critical embolic events, including disabling stroke, if the TAVR device is advanced into the ascending aorta without preventive measures. However, there are no previous reports describing these masses, including their incidence, predictors, and prognosis. Here we report our institutional experience with emboligenic matter (EM) and investigate the incidence and predictors of EM during TAVR, impact of EM on the procedure, and incidence of symptomatic ischemic stroke post procedure.

\section{Materials And Methods Study population}


This was a single-center prospective observational study that enrolled patients between May 2016 and October 2020. Among 436 consecutive patients with severe aortic valve stenosis (AS) or failing surgical aortic bioprostheses scheduled to undergo TAVR, 407 were evaluated. Twenty-two patients who underwent TAVR via a non-TF approach and 7 patients who underwent TF-TAVR under conscious sedation or local anesthesia without TEE, were excluded from analysis (Fig. 1). Data on laboratory findings (B-type natriuretic peptide [BNP] level) and echocardiographic parameters (aortic valve area [AVA], peak velocity [Vmax], mean aortic valve pressure gradient [mPG], and left ventricular ejection fraction [LVEF]) were collected. Echocardiographic parameters were obtained according to American Society of Echocardiography recommendations [5]. LVEF was estimated using the biplane Simpson method. This study complied with the Helsinki Declaration and was approved by the ethical committee of Kyoto Prefectural University of Medicine (Kyoto, Japan).

\section{Definition of abdominal aorta and iliofemoral artery tortuosity by computed tomography}

All patients underwent plain and contrast-enhanced computed tomography (CT) before TAVR, using a 320-slice system (Canon Aquilion ONE Vision Edition; Canon Medical Systems, Tochigi, Japan) with three-dimensional reconstruction software (Ziostation $2^{\circledR}$, Ziosoft, Tokyo, Japan). Surgical strategy and device selection were planned based on CT imaging information as previously reported [6]. CT scans were also used to screen for abdominal aorta and iliofemoral artery tortuosity, as previously described [7] A tortuous abdominal aorta was defined as the existence of a clear angulation, with an angle of $>45^{\circ}$, whereas a tortuous iliofemoral arterial axis corresponded to the existence of a clear angulation of $>90^{\circ}$. Abdominal aortic aneurysm (AAA) was identified with a diameter of $\geq 30$ $\mathrm{mm}$. The presence of mural thrombus was defined as a wall thickness $>2 \mathrm{~mm}$ in the descending thoracic or abdominal aorta [8].

\section{TF-TAVR procedure and definition of EM}

Indication for TAVR was determined based on the clinical consensus reached by our heart team, which consisted of cardiologists, cardiovascular surgeons, anaesthesiologists, and imaging specialists. All patients underwent TAVR using a balloon-expandable (SAPIEN XT or 3 transcatheter heart valve; Edwards Lifesciences, Irvine, CA, USA) or self-expandable prothesis (CoreValve or CoreValve Evolut R/PRO Revalving System; Medtronic, Minneapolis, MN, USA). TAVR was performed using the TF approach under general anesthesia and TEE (PHILIPS-EPIC7 or iE33 systems; Philips, Bothell, WA, USA) guidance. Heparin was administrated intravenously by the anaesthesiologist after inserting an 8Fr sheath and intraoperative activated clotting time (ACT) was maintained for more than $250 \mathrm{~s}$. The valve catheter was advanced to the descending thoracic aorta and just prior to entering the aortic arch the catheter tip was checked by TEE for any attached EM (Fig. 2a). EM was defined as a mobile membranous mass attached around the catheter tip, which can be confirmed only by TEE within the proximal part of the descending thoracic aorta (Fig. 2b and 2c and Supplemental Video 2), and resembles seaweed swaying in blood flow as we have reported [4].

\section{Anti-embolization preventive measures}

In cases of self-expandable valve implantation, if EM was detected we removed the entire device and restarted the procedure from device insertion. The catheter tip was grossly inspected, cleaned with saline, and reinserted with the entire catheter system or through an additional 18 or 20 French (Fr) outer sheath, depending on the condition of the patient's access root. The catheter tip was rechecked by TEE to confirm absence of EM and whether the catheter could be advanced to the ascending aorta for transcatheter heart valve (THV) implantation (Fig. 2d and 2e and 
Supplemental Video 3). At the end of the procedure, peripheral access route angiography was used to check if there were any access route vascular complications. Procedural success and complications were defined according to Valve Academic Research Consortium-2 (VARC-2) criteria.9 Of note, the procedure could not be restarted in cases of balloon-expandable valve implantation.

\section{End points and statistical analysis}

The primary end point of this study was incidence of symptomatic ischemic stroke within $24 \mathrm{~h}$ post procedure. The secondary end points were vascular access site and access-related complications according to VARC-2 criteria [9].

Non-normal continuous variables are presented as median (IQR), as were hemoglobin $(\mathrm{Hb})$ and minimum lumen diameter (MLD) of common femoral artery (CFA), which although normally distributed, had nearly identical median and mean values. Differences between groups were compared using Wilcoxon rank sum test, and paired $t$ test was used for $\mathrm{Hb}$ and MLD of CFA. Categorical variables are shown as frequencies (\%) and groups compared with Fisher exact test. Multiple regression analysis was conducted to identify independent determinants of EM among cases treated with a self-expandable prothesis. The following variables were considered as possible confounders:

comorbidity with peripheral artery disease (PAD), InLine sheath usage, MLD of iliac and CFA, tortuosity of aorta and iliac artery, and presence of aortic mural thrombus. Three clinically important variables with low $p$ values were included in the model. Cumulative incidence of major adverse cardiac and cerebrovascular events (MACCE), allcause death, heart failure rehospitalization, and ischemic stroke during the 30-day period post TAVR were also compared between groups. A p-value $<0.05$ was considered statistically significant. All statistical analyses were performed using JMP software (version 14.0; JMP, SAS Institute, Cary, NC, USA).

\section{Results}

\section{Incidence rate and comparison of baseline clinical characteristics between patients with or without EM}

Among 436 consecutive patients who were scheduled for TAVR, 407 were evaluated (Fig. 1) and a total of 15 cases (3.7\%) of EM were identified (Table 1). The prevalence of past history of coronary artery disease $(20.0 \%$ vs. $36.2 \%, p$ $=0.27)$ and cerebrovascular event $(0.00 \%$ vs. $16.1 \%, p=0.14)$ was lower and prevalence of PAD $(33.3 \%$ vs. $19.9 \%, p$ $=0.2$ ) was higher in the EM (+) group, but differences were not statistically significant. There were also no significant differences in other baseline clinical characteristics between groups. 
Table 1

Study patients' characteristics

\begin{tabular}{|c|c|c|c|c|}
\hline \multirow[t]{2}{*}{ Parameters } & All & $\mathrm{EM}(+)$ & EM (-) & \multirow[t]{2}{*}{ p-value } \\
\hline & $N=407$ & $N=15$ & $N=392$ & \\
\hline Age, years & $86(84-89)$ & $86(84-91)$ & $86(83-89)$ & 0.380 \\
\hline Male, n (\%) & $111(27.3)$ & $4(26.7)$ & 107 (27.3) & 1.000 \\
\hline $\mathrm{BMI}, \mathrm{kg} / \mathrm{m}^{2}$ & $21.1(19.0-23.9)$ & $20.5(17.6-22.2)$ & $21.2(19.1-24.0)$ & 0.140 \\
\hline STS score (\%) & $5.9(4.3-8.8)$ & $6.1(4.7-10.0)$ & $5.9(4.3-8.8)$ & 0.820 \\
\hline Frailty scale $\geq 6, n(\%)$ & $175(43.0)$ & $9(60.0)$ & $31(34.1)$ & 0.055 \\
\hline NYHA class III/IV, n (\%) & $135(33.2)$ & $3(20.0)$ & $132(33.7)$ & 0.400 \\
\hline Hypertension, n (\%) & $297(73.0)$ & $13(86.7)$ & $284(72.5)$ & 0.370 \\
\hline Dyslipidemia, n (\%) & $177(43.5)$ & $4(26.7)$ & $173(44.1)$ & 0.290 \\
\hline Diabetes mellitus, n (\%) & $90(22.1)$ & $3(20.0)$ & $87(22.2)$ & 1.000 \\
\hline PAD, n (\%) & $83(20.4)$ & $5(33.3)$ & 78 (19.9) & 0.200 \\
\hline CAD, n (\%) & $145(35.6)$ & $3(20.0)$ & $87(22.2)$ & 1.000 \\
\hline Prior CVA, n (\%) & $63(15.5)$ & $0(0.00)$ & $63(16.1)$ & 0.140 \\
\hline SVD, n (\%) & $8(1.97)$ & $1(6.67)$ & $7(1.79)$ & 0.260 \\
\hline \multicolumn{5}{|l|}{ Laboratory data } \\
\hline $\mathrm{Hb}, \mathrm{g} / \mathrm{dL}$ & $10.9(9.9-12.2)$ & $11.5(10.6-13.2)$ & $10.9(9.8-12.2)$ & 0.250 \\
\hline $\mathrm{Cr}, \mathrm{mg} / \mathrm{dL}$ & $0.90(0.69-1.20)$ & $0.84(0.70-0.96)$ & $0.91(0.70-1.20)$ & 0.230 \\
\hline BNP, pg/mL & $245(94-520)$ & $211(87-335)$ & $246(94-529)$ & 0.880 \\
\hline Alb, g/dL & $3.7(3.4-4.0)$ & $3.9(3.5-4.0)$ & $3.7(3.4-4.0)$ & 0.490 \\
\hline \multicolumn{5}{|l|}{ TTE data } \\
\hline Peak velocity, m/s & $4.4(4.1-5.0)$ & $4.4(4.2-5.1)$ & $4.4(4.1-5.0)$ & 0.680 \\
\hline Mean pressure gradient, mmHg & $47(39-60)$ & $49(39-67)$ & $47(39-59)$ & 0.470 \\
\hline AVA, $\mathrm{cm}^{2}$ & $0.6(0.5-0.7)$ & $0.5(0.4-0.6)$ & $0.6(0.5-0.7)$ & 0.110 \\
\hline LVEF, \% & $62(52-69)$ & $60(39-72)$ & $62(53-69)$ & 0.400 \\
\hline
\end{tabular}

Continuous and categorical variables are presented as median (25-75th percentile) and as number (percentage), respectively.

$\mathrm{AAA}=$ abdominal aortic aneurysm; Alb = albumin; $\mathrm{AVA}=$ aortic valve area; $\mathrm{BMI}=$ body mass index; $\mathrm{BNP}=\mathrm{B}$-type natriuretic peptide; $\mathrm{CAD}=$ coronary artery disease; $\mathrm{CFA}=$ common femoral artery; $\mathrm{Cr}=$ creatinine; $\mathrm{CT}=$ computed tomography; CVA = cerebrovascular event; $\mathrm{EM}=$ emboligenic matter; $\mathrm{Hb}=$ hemoglobin; $\mathrm{LVEF}=$ left ventricular ejection fraction; MLD = minimum lumen diameter; NYHA = New York Heart Association functional classification; PAD = peripheral artery disease; STS = Society of Thoracic Surgeons; SVD = structural valve deterioration 


\begin{tabular}{|c|c|c|c|c|}
\hline \multirow[t]{2}{*}{ Parameters } & All & EM (+) & EM (-) & \multirow[t]{2}{*}{ p-value } \\
\hline & $N=407$ & $N=15$ & $N=392$ & \\
\hline \multicolumn{5}{|l|}{ CT data } \\
\hline CFA-MLD, mm & $6.7(5.8-7.6)$ & $6.7(6.1-7.6)$ & $6.7(5.8-7.6)$ & 0.810 \\
\hline lliac artery MLD, mm & $5.8(5.0-6.7)$ & $6.1(5.3-6.6)$ & $5.8(5.0-6.7)$ & 0.530 \\
\hline Abdominal aorta tortuosity, $n$ (\%) & $185(45.5)$ & $7(46.7)$ & $178(45.4)$ & 1.000 \\
\hline lliac artery tortuosity, n (\%) & $165(40.5)$ & $5(33.3)$ & $160(40.8)$ & 0.790 \\
\hline AAA, n (\%) & $27(6.63)$ & $0(0.00)$ & $27(6.89)$ & 0.610 \\
\hline Mural thrombus, $\mathrm{n}(\%)$ & $183(45.0)$ & $10(66.7)$ & $173(44.1)$ & 0.110 \\
\hline \multicolumn{5}{|l|}{ Procedural data } \\
\hline Cut down, n (\%) & $49(12.0)$ & $0(0.00)$ & $49(12.5)$ & 0.230 \\
\hline Procedural success, n (\%) & $402(98.8)$ & $14(93.3)$ & $388(99.0)$ & 0.170 \\
\hline \multicolumn{5}{|l|}{ Valve type } \\
\hline Self-expandable valve, $n$ (\%) & $182(44.7)$ & $15(100.0)$ & $167(42.6)$ & $<0.0001$ \\
\hline Inline sheath, $\mathrm{n}(\%)$ & $121(29.7)$ & $14(93.3)$ & $107(27.3)$ & $<0.0001$ \\
\hline Outer sheath, n (\%) & $286(70.3)$ & $1(6.67)$ & $285(72.7)$ & $<0.0001$ \\
\hline \multicolumn{5}{|c|}{$\begin{array}{l}\text { Continuous and categorical variables are presented as median ( } 25-75 \text { th percentile) and as number } \\
\text { (percentage), respectively. }\end{array}$} \\
\hline \multicolumn{5}{|c|}{ 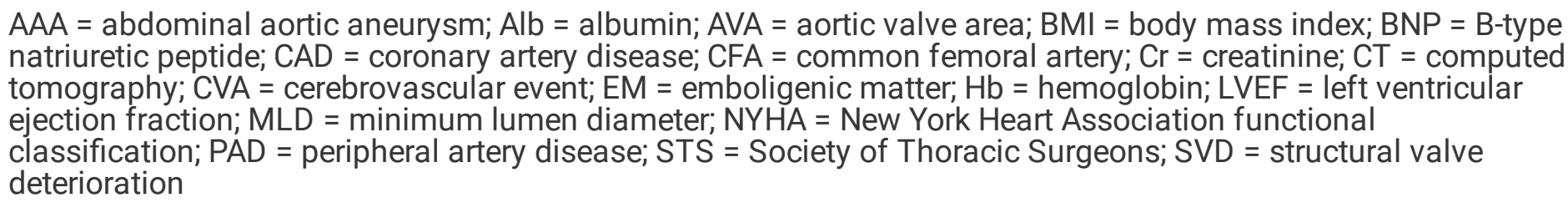 } \\
\hline
\end{tabular}

\section{Baseline laboratory, TTE, and CT comparison between patients with or without EM}

Laboratory, TTE, and CT results are presented in Table 1. Laboratory data did not reveal significant differences between groups. AS severity and LVEF were also similar. MLD of CFA did not vary $(6.7 \mathrm{~mm}$ vs. $6.7 \mathrm{~mm}, \mathrm{p}=0.81)$ but the iliac artery was larger $(6.1 \mathrm{~mm}$ vs. $5.8 \mathrm{~mm}, \mathrm{p}=0.53)$ in the EM $(+)$ group. Abdominal aorta tortuosity was more frequent $(46.7 \%$ vs. $45.4 \%, p=1.0)$, but iliac artery tortuosity less frequent $(33.3 \%$ vs. $40.8 \%, p=0.79)$ in the EM $(+)$ group. There were no AAA cases in the EM (+) group and the incidence of mural thrombus existence was higher $(66.7 \%$ vs. $44.1 \%, p=0.11)$ in that group. However, none of these differences were statistically significant.

\section{Comparisons of TAVR procedure results between patients with or without EM}

As shown in Table 1, the overall procedural success rate defined according to VARC-2 criteria [9] was $98.8 \%$ and there was no significant difference between groups (93.3\% vs. 99.0\%, $p=0.17)$. There were no surgical cut down procedures required in the EM (+) group. A self-expandable valve was used more often in the EM (+) group (100\% 
vs. $42.6 \%$, p < 0.0001). Fourteen of these cases used the CoreValve's InLine sheath system $(93.3 \%$ vs. $27.3 \%, p<$ $0.0001)$ and the other case used an outer sheath $(6.67 \%$ vs. $72.7 \%, p<0.0001)$ initially.

\section{Ischemic stroke, vascular complications and 30-day MACCE}

Results on ischemic stroke, vascular complications and 30-day MACCE are presented in Table 2. The overall ischemic stroke rate within $24 \mathrm{~h}$ post TAVR was $1.97 \%$, with no cases in the EM (+) group. Non-cerebral distal embolization did not occur in patients within the EM (+) group, but one case occurred in the EM (-) group $(0.00 \%$ vs. $0.26 \%, p=1.00)$. Two cases of new ipsilateral lower extremity ischemia occurred in patients within the EM (+) group, with three cases in the other group $(13.3 \%$ vs. $0.77 \%, p=0.01)$. The number of major vascular complications defined according to VARC-2 criteria [9] was higher in the EM (+) group (13.3\% vs. 5.10\%, p = 0.19), but not significantly. Minor vascular complications defined according to VARC-2 criteria [9] occurred more frequently in the EM (+) group $(20.0 \%$ vs. $4.08 \%, p=0.028)$. No MACCE events within 30 days post TAVR occurred in patients within the EM (+) group, but 18 cases occurred in the EM (-) group ( $0.00 \%$ vs. $4.59 \%, p=1.00$; Table 2$)$, though the difference was not statistically significant. 


\begin{tabular}{|c|c|c|c|c|}
\hline \multirow[t]{2}{*}{ Parameters } & All & $\operatorname{EM}(+)$ & $\mathrm{EM}(-)$ & \multirow{2}{*}{$\begin{array}{l}\mathrm{p}- \\
\text { value }\end{array}$} \\
\hline & $N=407$ & $N=15$ & $N=392$ & \\
\hline \multicolumn{5}{|l|}{ Primary outcome } \\
\hline Ischemic stroke, $n$ (\%) & $8(1.97)$ & $0(0.00)$ & $8(2.04)$ & 1.000 \\
\hline \multicolumn{5}{|l|}{ Secondary outcome } \\
\hline Major vascular complications, n (\%) & $22(5.41)$ & $2(13.3)$ & $20(5.10)$ & 0.190 \\
\hline Aortic dissection, $\mathrm{n}(\%)$ & $5(1.23)$ & $0(0.00)$ & $5(1.28)$ & 1.000 \\
\hline Annulus rupture, $\mathrm{n}(\%)$ & $2(0.49)$ & $0(0.00)$ & $2(0.51)$ & 1.000 \\
\hline Left ventricle perforation, $n$ (\%) & $2(0.49)$ & $0(0.00)$ & $2(0.51)$ & 1.000 \\
\hline Access site or access-related vascular injury, $n(\%)$ & $7(1.72)$ & $0(0.00)$ & $7(1.79)$ & 1.000 \\
\hline Distal embolization (non-cerebral), $\mathrm{n}(\%)$ & $1(0.25)$ & $0(0.00)$ & $1(0.26)$ & 1.000 \\
\hline New ipsilateral lower extremity ischemia, n (\%) & $5(1.23)$ & $2(13.3)$ & $3(0.77)$ & 0.0119 \\
\hline Minor vascular complications, n (\%) & $19(4.67)$ & $3(20.0)$ & $16(4.08)$ & 0.0275 \\
\hline Access site or access-related vascular injury, $n(\%)$ & $6(1.55)$ & $1(7.14)$ & $5(1.34)$ & 0.200 \\
\hline Unplanned endovascular stenting, $\mathrm{n}(\%)$ & $2(0.49)$ & $1(6.67)$ & $1(0.26)$ & 0.072 \\
\hline Vascular repair, n (\%) & $11(2.70)$ & $1(6.67)$ & $10(2.55)$ & 0.340 \\
\hline Percutaneous closure device failure, $n$ (\%) & $10(2.46)$ & $1(6.67)$ & $9(2.30)$ & 0.320 \\
\hline 30-day MACCE, n (\%) & $18(4.42)$ & $0(0.00)$ & $18(4.59)$ & 1.000 \\
\hline All-cause death, $\mathrm{n}(\%)$ & $7(1.72)$ & $0(0.00)$ & $7(1.79)$ & 1.000 \\
\hline Heart failure rehospitalization, $\mathrm{n}(\%)$ & $1(0.25)$ & $0(0.00)$ & $1(0.26)$ & 1.000 \\
\hline Ischemic stroke, $\mathrm{n}(\%)$ & $12(2.95)$ & $0(0.00)$ & $12(3.06)$ & 1.000 \\
\hline \multicolumn{5}{|c|}{ Categorical variables are presented as number (percentage). } \\
\hline $\mathrm{EM}=$ emboligenic matter; $\mathrm{MACCE}=$ major adverse & ular and & יםc0 & rents & \\
\hline
\end{tabular}

\section{Univariate and multivariate analyses of the predictors of EM}

The CoreValve's InLine sheath was strongly correlated with EM. Univariate and multivariate analysis were performed to compare the predictors of EM in the self-expandable valve group. In univariate analysis (Table 3 ), InLine sheath usage was associated with EM (OR 7.85; 95\% Cl 1.00-61.2; $\mathrm{p}=0.0492)$. In multivariate analysis (Table 4), the following variables were included: InLine sheath usage, MLD of the iliac artery, and tortuosity of the iliac artery (model 1); PAD, InLine sheath usage, and MLD of the iliac artery (model 2); InLine sheath usage, MLD of the iliac artery, and MLD of the CFA (model 3). In all models, InLine sheath usage was identified as an independent predictor of EM (OR 8.14; 95\% Cl 1.04-63.8; $p=0.0459$ [model 1], OR 9.59; 95\% Cl 1.19-77.2; $p=0.0337$ [model 2], and OR 8.12; $95 \% \mathrm{Cl} 1.03-63.7 ; p=0.0464$ [model 3]). 
Table 3

Univariate logistic regression analyses of the predictors of causing emboligenic matter

\begin{tabular}{|llll|}
\hline Parameters & OR & $95 \% \mathrm{Cl}$ & p-value \\
\hline PAD & 1.4 & $0.45-4.31$ & 0.560 \\
\hline Inline sheath & 7.85 & $1.00-61.2$ & 0.0492 \\
\hline Iliac artery MLD & 1.29 & $0.80-2.07$ & 0.290 \\
CFA-MLD & 1.17 & $0.78-1.75$ & 0.460 \\
\hline Iliac artery tortuosity & 0.85 & $0.28-2.59$ & 0.770 \\
\hline Aorta tortuosity & 0.95 & $0.33-2.74$ & 0.930 \\
\hline Mural thrombus & 2.28 & $0.75-6.96$ & 0.150 \\
\hline Cl = confidence interval; MLD = minimal lumen diameter; OR = odds ratio; PAD = peripheral artery disease \\
\hline
\end{tabular}

Table 4

Multivariate logistic regression analyses of the predictors of causing emboligenic matter

\begin{tabular}{|c|c|c|c|c|c|c|c|c|c|}
\hline \multirow[b]{2}{*}{ Parameters } & \multicolumn{3}{|c|}{ Model 1} & \multicolumn{3}{|c|}{ Model 2} & \multicolumn{3}{|c|}{ Model 3} \\
\hline & OR & $95 \% \mathrm{Cl}$ & $\begin{array}{l}\mathrm{p}- \\
\text { value }\end{array}$ & OR & $95 \% \mathrm{Cl}$ & $\begin{array}{l}\mathrm{p}- \\
\text { value }\end{array}$ & OR & $95 \% \mathrm{Cl}$ & $\begin{array}{l}\mathrm{p}- \\
\text { value }\end{array}$ \\
\hline PAD & & & & 2.12 & $\begin{array}{l}0.64- \\
7.03\end{array}$ & 0.230 & & & \\
\hline InLine sheath & 8.14 & $\begin{array}{l}1.04- \\
63.8\end{array}$ & 0.0459 & 9.59 & $\begin{array}{l}1.19- \\
77.2\end{array}$ & 0.0337 & 8.12 & $\begin{array}{l}1.03- \\
63.7\end{array}$ & 0.0464 \\
\hline Iliac artery MLD & 0.73 & $\begin{array}{l}0.44- \\
1.21\end{array}$ & 0.220 & 0.71 & $\begin{array}{l}0.43- \\
1.18\end{array}$ & 0.190 & 0.76 & $\begin{array}{l}0.40- \\
1.45\end{array}$ & 0.4000 \\
\hline CFA-MLD & & & & & & & 0.99 & $\begin{array}{l}0.57- \\
1.71\end{array}$ & 0.970 \\
\hline $\begin{array}{l}\text { lliac artery } \\
\text { tortuosity }\end{array}$ & 0.75 & $\begin{array}{l}0.23- \\
2.43\end{array}$ & 0.640 & & & & & & \\
\hline \multicolumn{10}{|l|}{ Aorta tortuosity } \\
\hline \multicolumn{10}{|l|}{ Mural thrombus } \\
\hline
\end{tabular}

\section{Cases positive for EM and representative case}

All EM (+) cases are described in Table 5. lliac artery MLD ranged from $4.1 \mathrm{~mm}$ to $7.9 \mathrm{~mm}$ and CFA-MLD from 4.2 $\mathrm{mm}$ to $9.7 \mathrm{~mm}$. EM size measured by TEE varied from $1.2 \mathrm{~mm}$ to $7.2 \mathrm{~mm}$. No EM (+) case experienced ischemic stroke or symptomatic non-cerebral distal embolism, having undertaken preventive measures as previously described. Nine cases did not require an additional outer sheath but six cases did, to prevent THV device contact with the vessel wall of the access root in the lower limb and abdominal aorta. Images (Fig. 2) and movie (Supplemental Video 2) of EM in Case 14 are shown. 
Table 5

Clinical summaries of the 15 emboligenic matter case

\begin{tabular}{|c|c|c|c|c|c|c|c|c|c|}
\hline Case & Age & Sex & $\begin{array}{l}\text { lliac- } \\
\text { MLD } \\
(\mathrm{mm})\end{array}$ & $\begin{array}{l}\text { CFA- } \\
\text { MLD } \\
(\mathrm{mm})\end{array}$ & $\begin{array}{l}\text { Size of } \\
\text { emboligenic } \\
\text { matter } \\
(\mathrm{mm})\end{array}$ & $\begin{array}{l}\text { How to } \\
\text { bail out }\end{array}$ & $\begin{array}{l}\text { Number } \\
\text { of } \\
\text { times } \\
\text { to } \\
\text { reinsert }\end{array}$ & Complications & $\begin{array}{l}\text { Ischemic } \\
\text { stroke }\end{array}$ \\
\hline 1 & 85 & Female & 4.1 & 5.9 & 3.2 & $\begin{array}{l}\text { Inserted } \\
\text { an } \\
\text { additional } \\
\text { outer } \\
\text { sheath }\end{array}$ & 1 & $\begin{array}{l}\text { Iliac artery } \\
\text { dissection }\end{array}$ & No \\
\hline 2 & 87 & Female & 5.0 & 4.2 & 2.2 & $\begin{array}{l}\text { Reinserted } \\
\text { InLine } \\
\text { sheath }\end{array}$ & 1 & CFA occlusion & No \\
\hline 3 & 84 & Male & 6.6 & 7.6 & 5.6 & $\begin{array}{l}\text { Reinserted } \\
\text { InLine } \\
\text { sheath }\end{array}$ & 1 & No & No \\
\hline 4 & 94 & Female & 5.3 & 6.0 & 9.0 & $\begin{array}{l}\text { Inserted } \\
\text { an } \\
\text { additional } \\
\text { outer } \\
\text { sheath }\end{array}$ & 1 & $\begin{array}{l}\text { Iliac artery } \\
\text { dissection }\end{array}$ & No \\
\hline 5 & 85 & Female & 4.9 & 6.7 & 2.7 & $\begin{array}{l}\text { Reinserted } \\
\text { InLine } \\
\text { sheath }\end{array}$ & 1 & No & No \\
\hline 6 & 91 & Female & 4.5 & 5.4 & 2.9 & $\begin{array}{l}\text { Inserted } \\
\text { an } \\
\text { additional } \\
\text { outer } \\
\text { sheath }\end{array}$ & 1 & $\begin{array}{l}\text { Haemostatic } \\
\text { trouble }\end{array}$ & No \\
\hline 7 & 83 & Female & 7.9 & 8.5 & 5.0 & $\begin{array}{l}\text { Inserted } \\
\text { an } \\
\text { additional } \\
\text { outer } \\
\text { sheath }\end{array}$ & 1 & No & No \\
\hline 8 & 86 & Female & 6.5 & 6.1 & 2.1 & $\begin{array}{l}\text { Reinserted } \\
\text { InLine } \\
\text { sheath }\end{array}$ & 1 & No & No \\
\hline 9 & 92 & Female & 6.1 & 6.2 & 2.8 & $\begin{array}{l}\text { Reinserted } \\
\text { InLine } \\
\text { sheath }\end{array}$ & 1 & No & No \\
\hline 10 & 93 & Male & 5.3 & 6.7 & 1.2 & $\begin{array}{l}\text { Reinserted } \\
\text { InLine } \\
\text { sheath }\end{array}$ & 1 & No & No \\
\hline 11 & 82 & Male & 7.9 & 9.7 & 4.8 & $\begin{array}{l}\text { Reinserted } \\
\text { InLine } \\
\text { sheath }\end{array}$ & 1 & No & No \\
\hline
\end{tabular}

$\mathrm{CFA}=$ common femoral artery; MLD = minimal lumen diameter 


\begin{tabular}{|c|c|c|c|c|c|c|c|c|c|}
\hline Case & Age & Sex & $\begin{array}{l}\text { lliac- } \\
\text { MLD } \\
(\mathrm{mm})\end{array}$ & $\begin{array}{l}\text { CFA- } \\
\text { MLD } \\
(\mathrm{mm})\end{array}$ & $\begin{array}{l}\text { Size of } \\
\text { emboligenic } \\
\text { matter } \\
(\mathrm{mm})\end{array}$ & $\begin{array}{l}\text { How to } \\
\text { bail out }\end{array}$ & $\begin{array}{l}\text { Number } \\
\text { of } \\
\text { times } \\
\text { to } \\
\text { reinsert }\end{array}$ & Complications & $\begin{array}{l}\text { Ischemic } \\
\text { stroke }\end{array}$ \\
\hline 12 & 90 & Female & 6.1 & 7.0 & 2.9 & $\begin{array}{l}\text { Reinserted } \\
\text { InLine } \\
\text { sheath }\end{array}$ & 2 & No & No \\
\hline 13 & 91 & Female & 6.8 & 6.5 & 1.7 & $\begin{array}{l}\text { Reinserted } \\
\text { InLine } \\
\text { sheath }\end{array}$ & 1 & No & No \\
\hline 14 & 79 & Female & 5.5 & 6.1 & 7.2 & $\begin{array}{l}\text { Inserted } \\
\text { an } \\
\text { additional } \\
\text { outer } \\
\text { sheath }\end{array}$ & 2 & $\begin{array}{l}\text { lliac artery } \\
\text { dissection }\end{array}$ & No \\
\hline 15 & 86 & Male & 6.4 & 9.5 & 5.5 & $\begin{array}{l}\text { Inserted } \\
\text { an } \\
\text { additional } \\
\text { outer } \\
\text { sheath }\end{array}$ & 2 & No & No \\
\hline
\end{tabular}

\section{Representative case}

A 79-year-old female who underwent surgical aortic valve replacement with Trifecta (St Jude Medical Inc., St Paul, MN, USA) was admitted to our hospital for acute heart failure. Echocardiography revealed severe AS associated with significant regurgitation secondary to prosthesis malfunction (aortic valve area: $0.4-\mathrm{cm}^{2}$; mean gradient: 95 $\mathrm{mmHg}$ ). TAVR using a self-expandable CoreValve Evolut $\mathrm{R}$ valve was performed via the CFA under TEE guidance. After advancing the CoreValve system to the descending thoracic aorta we checked the catheter tip using TEE (Fig. 3a), which revealed a mobile membranous mass (Fig. 3b and 3c). After removing the CoreValve system, we confirmed the membranous mass attached to the catheter. After clearing the mass from the catheter, it was reinserted. Repeat TEE revealed another mass was attached. We therefore removed the system and cleared the mass again, before inserting an additional 18-Fr outer sheath and passing the device through it. Subsequent TEE demonstrated absence of a catheter tip mass (Fig. 3d and 3e); therefore, we advanced the system through the aortic arch. The valve was successfully implanted. Thereafter, access route angiography revealed dissection of the external iliac artery (Fig. 3f) and intravascular ultrasound revealed intimal exfoliation (Fig. 3g and Supplemental Video 4). Conservative treatment was chosen because there was no flow delay.

\section{Discussion}

In this study, EM was found in 3.7\% of patients during TF-TAVR and all involved self-expandable THV implantation (Central illustration). In addition, most cases used the CoreValve's InLine sheath system. There was no symptomatic critical distal embolism including ischemic stroke, presumably avoided by restarting the procedure after cleaning the catheter tip with saline or using an outer sheath if needed. Vascular access complications, especially for the lower extremity, should be checked and managed with caution.

\section{The origin of EM}


The CoreValve's InLine sheath is integrated with the Evolut delivery catheter system. Generally, after a previously inserted 14- or 16- Fr sheath is removed, the CoreValve's InLine sheath is inserted, which means the CoreValve's InLine sheath system is sheathless, representing a naked THV insertion device. As the CoreValve's InLine sheath is advanced through the iliofemoral region, it may cause exfoliation of plaque, mural thrombus, or endothelium. The exfoliated material may attach to the advancing catheter tip on its way to the descending thoracic aorta. A previous report identified the material as endoarterial fibrous tissue based on pathological analysis [4]. The intraoperative ACT was confirmed to be greater than $250 \mathrm{~s}$ as appropriate, and the possibility of fibrin thrombus was very low. As a result, vascular complications including ipsilateral lower limb ischemia or access site or access-related vascular injury frequently occur presumably due to mobile masses originating from vascular tissue. However, the tortuosity or lumen size of the access vessel did not strongly correlate with EM, which suggests lower limb access lumen size is not large enough for the CoreValve's InLine sheath system to advance safely within the vessel. Only one case demonstrated EM during TAVR with 14-Fr eSheath (Edwards Lifesciences, Irvine, CA, USA) midway to the terminal aorta. In this case, the EM disappeared and TAVR was successfully completed with full insertion of 14-Fr eSheath after upgrading delivery guidewire to the more supportive Lunderkist (Cook Medical, Bloomington, IN, USA) as described previously [10]. In this case, the EM could have been produced after the THV device tip scratched the terminal aorta vessel wall. TAVR using an outer sheath, which included all balloon-expandable and most selfexpandable THV with a non-Inline sheath, revealed no EM in this study. This finding further supports the hypothesis that EM originates from the vascular wall tissue of access site vessels. Of note, EM size varied and most were quite small based on TEE estimations. Therefore, EM was not found in most cases when the device was removed, hence pathological evaluation for all cases was not possible.

\title{
Prevention of critical embolic complication
}

Ischemic stroke is a critical embolic complication after TAVR and its incident rate was reported as $2.3 \%$ at day 30 [11]. Even if asymptomatic, the frequency and burden of microembolization and cerebral ischemic injury may have a substantial impact on mid- and long-term cognitive function [12]. In this study, there were no symptomatic ischemic stroke events in 15 consecutive EM (+) cases at day 30 , having used preventive measures. Ischemic stroke could possibly be avoided by changing to the outer sheath system considering the proposed EM origin previously mentioned, and with no events in the nine cases who did not use the outer sheath. This result indicates that symptomatic ischemic strokes might be avoided by confirming the absence of EM by TEE or retrying with the CoreValve's InLine sheath system. However, although in some cases the small access root or its calcification precludes its use, outer sheaths larger than the CoreValve's InLine sheath might increase vascular complication incidence [13]. It is also cost-effective not to use larger sized outer sheaths. Thus, the strategy of CoreValve's InLine sheath reinsertion after clearing the EM with saline should be considered the first-line preventive measure against symptomatic ischemic stroke. Further, alternate management should be considered if absence of EM cannot be confirmed even after several reinsertions of the THV device. Ischemic stroke events after TAVR are associated with technical and procedural factors such as retrograde crossing of the stenotic aortic valve, catheter manipulation in an atherosclerotic aortic arch, and balloon aortic valvuloplasty [14]. However, the association with EM remains unclear because there are no reports describing this phenomenon. Notwithstanding, this study suggests that neurological outcomes might be improved in cases of self-expandable THV implantation with the CoreValve's InLine sheath system. In terms of other symptomatic peripheral artery embolic events, only one case of ipsilateral superficial femoral artery embolism was observed, which was rescued by endovascular treatment. Perioperative management considering the possibility of peripheral artery embolism from the subclavian, superior mesenteric, renal or lower extremity arteries and access root injury, should be executed carefully in EM positive cases.

\section{Role of TEE in the minimalist approach era}

\author{
Page $13 / 18$
}


EM was observed only in cases of CoreValve's InLine sheath use and was detected only by TEE within the descending aorta. According to the FORWARD study [15], CoreValve's InLine sheath system was used in $88.7 \%$ of cases. Considering these findings, we propose TEE may be useful for routine identification of EM in cases that use the CoreValve's InLine sheath system, even in the era of minimalist TAVR and fast-track TAVR courses.

\section{Limitations}

The present study has some limitations. First, the preventive measure had to be performed in all EM positive cases for ethical reasons, which prevented direct comparison with or without preventive measures. Second, the singlecenter design and Japanese sample may have introduced selection bias.

In conclusion, EM attached to the device tip was found by TEE within the proximal part of the descending thoracic aorta in $3.7 \%$ of TF-TAVR cases in this study but no ischemic stroke occurred in the EM (+) group. All EM (+) cases used a self-expandable valve while most used a CoreValve's InLine sheath system initially. The CoreValve's InLine sheath usage was identified as the only independent predictor of EM.

\section{Declarations}

\section{Acknowledgments: None}

\section{Authors' contributions:}

All authors contributed to the study conception and design. Masaki Yashige collected data, performed statistical analysis, and wrote the manuscript. Kan Zen, Michiyo Yamano, Tetsuhiro Yamano, Takeshi Nakamura, Hitoshi Yaku, and Satoaki Matoba revised the manuscript and figures. Shunsuke Nakamura, Tomotaka Fujimoto, Kazuaki Takamatsu, Nobuyasu Ito, and Yoshito Kadoya collected data and revised the manuscript. All authors read and approved the final manuscript.

Funding: This research did not receive any specific grant from funding agencies in the public, commercial, or notfor-profit sectors.

Conflict of Interest: The authors declare that they have no conflict of interest.

Ethics Approval: Approval was obtained from the ethics committee of Kyoto Prefectural University of Medicine. The procedures used in this study adhere to the tenets of the Declaration of Helsinki.

Consent to Participate: Informed consent was obtained from all individual participants included in the study.

\section{References}

1. Babaliaros V, Devireddy C, Lerakis S, Leonardi R, Iturra SA, Mavromatis K, Leshnower BG, Guyton RA, Kanitkar $\mathrm{M}$, Keegan P, Simone A (2014) Comparison of transfemoral transcatheter aortic valve replacement performed in the catheterization laboratory (minimalist approach) versus hybrid operating room (standard approach): outcomes and cost analysis. JACC Cardiovasc Interv 7:898-904. https://doi.org/10.1016/j.jcin.2014.04.005

2. Gurevich S, Oestreich B, Kelly RF, Mbai M, Bertog S, Ringsred K, Lawton A, Thooft B, Wagner J, Garcia S (2018) Outcomes of transcatheter aortic valve replacement using a minimalist approach. Cardiovasc Revasc Med 19:192-195. https://doi.org/10.1016/j.carrev.2017.08.002 
3. Butala NM, Chung M, Secemsky EA, Manandhar P, Marquis-Gravel G, Kosinski AS, Vemulapalli S, Yeh RW, Cohen DJ (2020) Conscious sedation versus general anesthesia for transcatheter aortic valve replacement: variation in practice and outcomes. JACC Cardiovasc Interv 13:1277-1287.

https://doi.org/10.1016/j.jcin.2020.03.008

4. Kadoya Y, Zen K, Yamano M, Yamano T, Nakamura T, Yaku H, Matoba S (2020) Exfoliated endothelium identified by transesophageal echocardiography during TAVR. JACC Cardiovasc Interv 13:e49-e50. https://doi.org/10.1016/j.jcin.2019.12.004

5. Lang RM, Bierig M, Devereux RB, Flachskampf FA, Foster E, Pellikka PA, Picard MH, Roman MJ, Seward J, Shanewise JS, Solomon SD (2005) Recommendations for chamber quantification: a report from the American Society of Echocardiography's Guidelines and Standards Committee and the Chamber Quantification Writing Group, developed in conjunction with the European Association of Echocardiography, a branch of the European Society of Cardiology. J Am Soc Echocardiogr 18:1440-1463. https://doi.org/10.1016/j.echo.2005.10.005

6. Blanke P, Weir-McCall JR, Achenbach S, Delgado V, Hausleiter J, Jilaihawi H, Marwan M, Nørgaard BL, Piazza N, Schoenhagen P, Leipsic JA (2019) Computed tomography imaging in the context of transcatheter aortic valve implantation (TAVI)/transcatheter aortic valve replacement (TAVR): An expert consensus document of the Society of Cardiovascular Computed Tomography. JACC Cardiovasc Imaging 12:1-24.

https://doi.org/10.1016/j.jcmg.2018.12.003

7. Kinnel M, Faroux L, Villecourt A, Tassan-Mangina S, Heroguelle V, Nazeyrollas P, Poncet A, Ruggieri VG, Metz D (2020) Abdominal aorta tortuosity on computed tomography identifies patients at risk of complications during transfemoral transcatheter aortic valve replacement. Arch Cardiovasc Dis 113:159-167.

https://doi.org/10.1016/j.acvd.2019.10.006

8. Roos CJ, Witkowska AJ, de Graaf MA, Veltman CE, Delgado V, de Grooth GJ, Jukema JW, Bax JJ, Scholte AJ (2013) Association of atherosclerosis in the descending thoracic aorta with coronary artery disease on multi detector row computed tomography coronary angiography in patients with suspected coronary artery disease. Int J Cardiovasc Imaging 29:1829-1837. https://doi.org/10.1007/s10554-013-0266-y

9. Kappetein AP, Head SJ, Généreux P, Piazza N, van Mieghem NM, Blackstone EH, Brott TG, Cohen DJ, Cutlip DE, Van Es GA, Hahn RT (2012) Updated standardized endpoint definitions for transcatheter aortic valve implantation: the Valve Academic Research Consortium-2 consensus document. Eur Heart J 33:2403-2418. https://doi.org/10.1093/eurheartj/ehs255

10. Yashige M, Zen K, Kadoya Y, Matoba S (2021) Combining the CoreValve Evolut PRO and 14-French eSheath in transfemoral transcatheter aortic valve replacement. Cardiovasc Interv Ther 36:266-267.

https://doi.org/10.1007/s12928-020-00659-1

11. Huded CP, Tuzcu EM, Krishnaswamy A, Mick SL, Kleiman NS, Svensson LG, Carroll J, Thourani VH, Kirtane AJ, Manandhar P, Kosinski AS (2019) Association between transcatheter aortic valve replacement and early postprocedural stroke. JAMA 321:2306-2315. https://doi.org/10.1001/jama.2019.7525

12. Mastoris I, Schoos MM, Dangas GD, Mehran R (2014) Stroke after transcatheter aortic valve replacement: incidence, risk factors, prognosis, and preventive strategies. Clin Cardiol 37:756-764. https://doi.org/10.1002/clc.22328

13. Toggweiler S, Gurvitch R, Leipsic J, Wood DA, Willson AB, Binder RK, Cheung A, Ye J, Webb JG (2012) Percutaneous aortic valve replacement: vascular outcomes with a fully percutaneous procedure. J Am Coll Cardiol 59:113-118. https://doi.org/10.1016/j.jacc.2011.08.069 
14. Giustino G, Mehran R, Veltkamp R, Faggioni M, Baber U, Dangas GD (2016) Neurological outcomes with embolic protection devices in patients undergoing transcatheter aortic valve replacement: a systematic review and meta-analysis of randomized controlled trials. JACC Cardiovasc Interv 9:2124-2133.

https://doi.org/10.1016/j.jcin.2016.07.024

15. Grube E, Van Mieghem NM, Bleiziffer S, Modine T, Bosmans J, Manoharan G, Linke A, Scholtz W, Tchétché D, Finkelstein A, Trillo R (2017) Clinical outcomes with a repositionable self-expanding transcatheter aortic valve prosthesis: The International FORWARD Study. J Am Coll Cardiol 70:845-853

\section{Figures}

\section{All patients who underwent TAVR at our institution between May 2016 to October 2020 $(n=436)$}

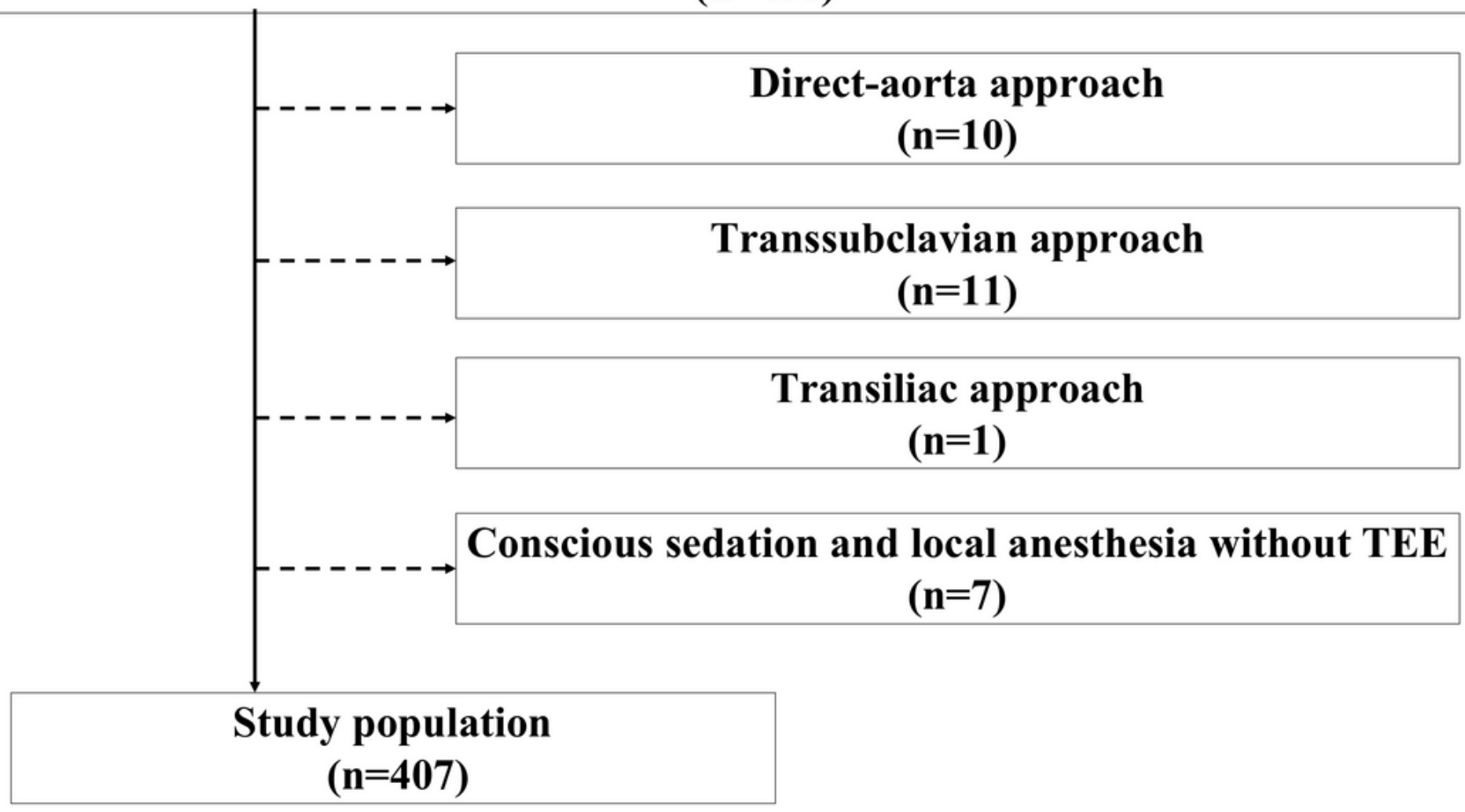

Figure 1

Flowchart depicting patient selection TAVR, transcatheter aortic valve replacement; TTE, transthoracic echocardiography 


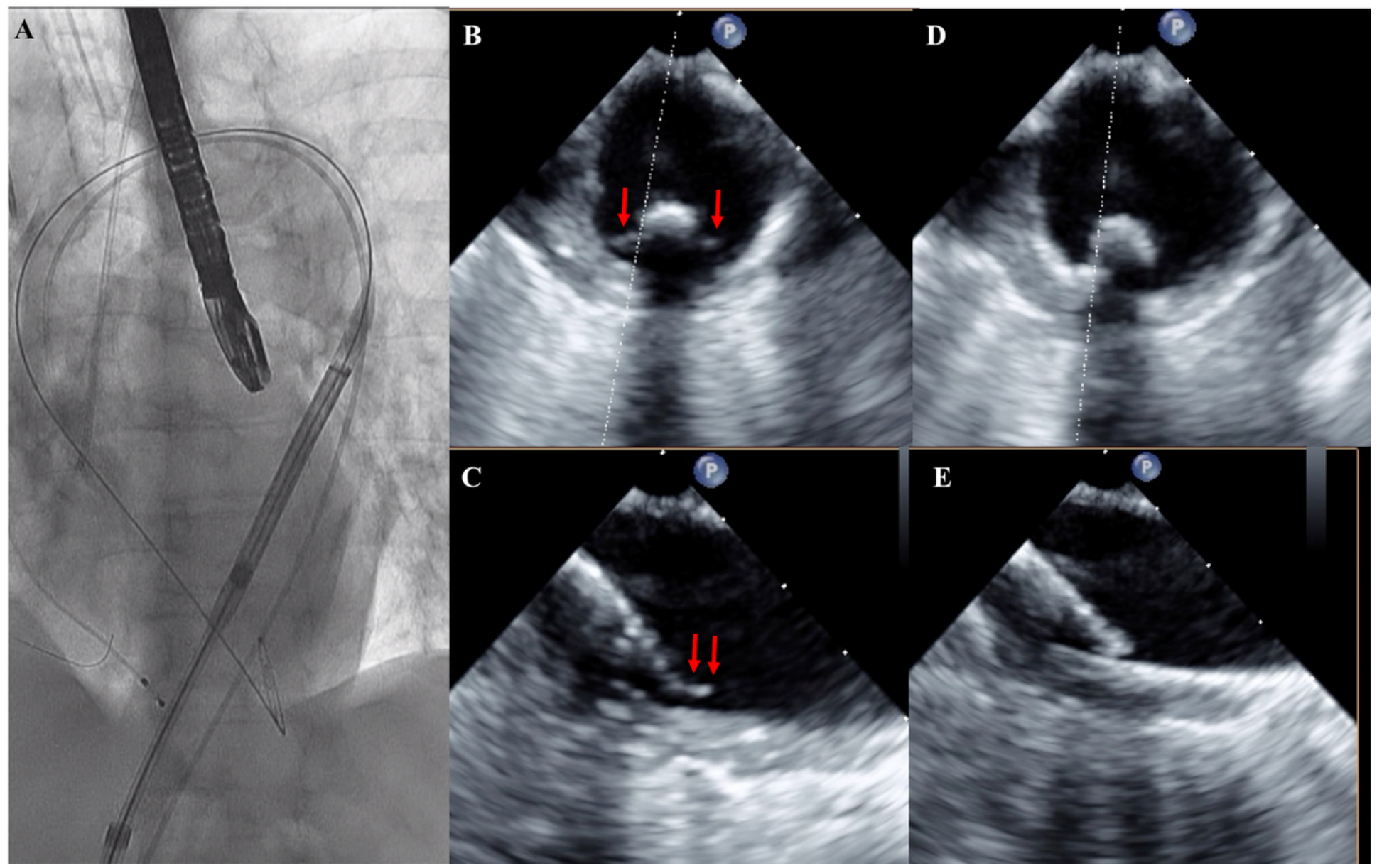

Figure 2

TF-TAVR procedure and definition of emboligenic matter a: Intraoperative TEE assessment of the descending aorta. $b$ and $c$ : TEE image of mobile membranous mass attached around catheter tip (red arrows); b: short axis, c: long axis. $d$ and e: TEE image after catheter reinserted; $d$ : short axis, e: long axis TF-TAVR, transfemoral transcatheter aortic valve replacement; TTE, transthoracic echocardiography
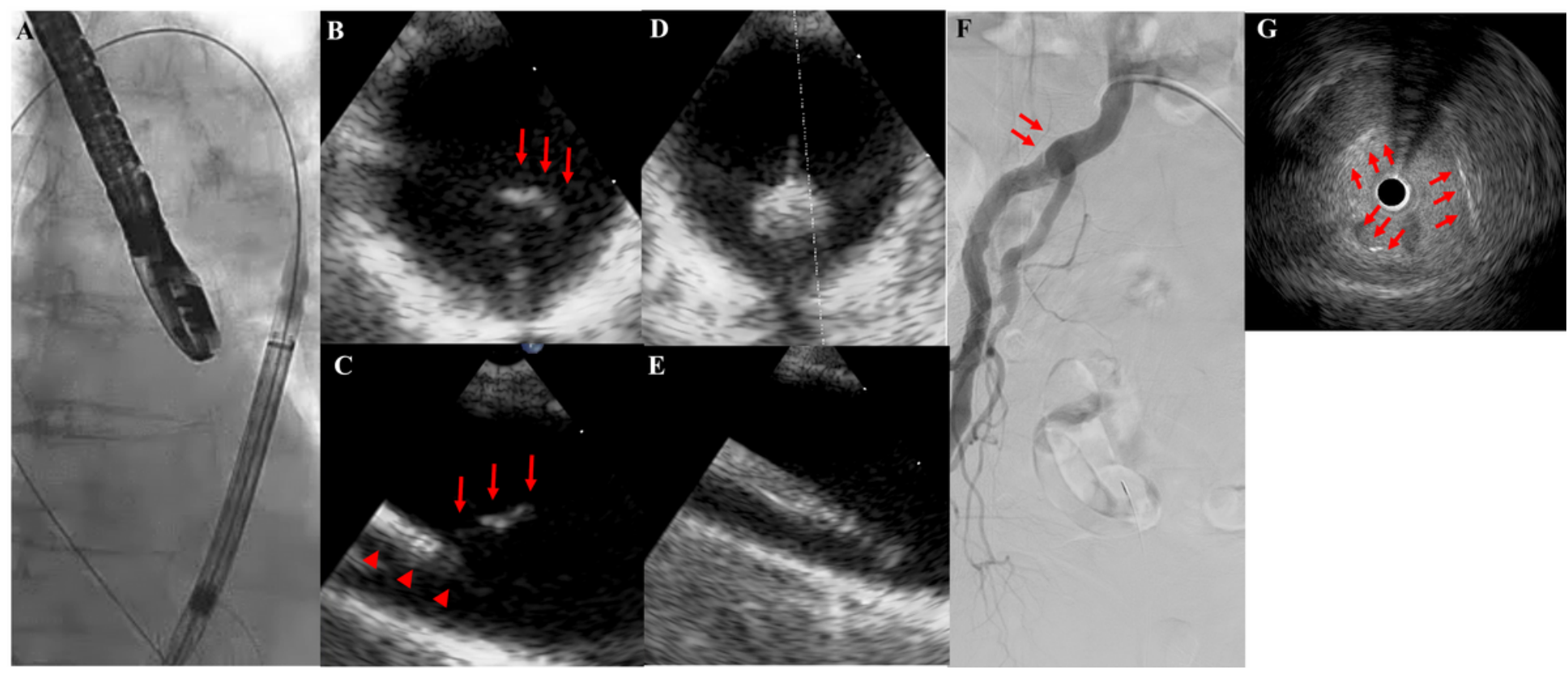

Figure 3 
Representative case: 79-year-old female with severe aortic stenosis due to prosthesis malfunction a: Intraoperative TEE assessment of the descending aorta. $b$ and $c$ : TEE showing mobile membranous mass (red arrows) at catheter tip (red arrowheads); b: short axis, c: long axis. $d$ and e: After CoreValve system reinserted, TEE showing mobile membranous mass removed; d: short axis, e: long axis. f: Angiography showing EIA dissection (red arrows). g: Intravascular ultrasound showing intimal exfoliation of the EIA (red arrows) TEE, transesophageal echocardiography; EIA, external iliac artery

\section{Supplementary Files}

This is a list of supplementary files associated with this preprint. Click to download.

- SupplementaryVideo1.mp4

- SupplementaryVideo2.mp4

- SupplementaryVideo3.mp4

- SupplementaryVideo4.mp4 